\title{
A CLASS OF STATIONARY PROCESSES AND A CENTRAL LIMIT THEOREM
}

\author{
By J. R. Blum and Murray Rosenblatt
}

1. Summary. A certain class of stationary processes is discussed. It is shown that each process in the class has an absolutely continuous spectrum. Under some moment conditions, it is shown that such processes satisfy the central limit theorem.

2. Introduction. Let $\eta=\left(\cdots, \eta_{-1}, \eta_{0}, \eta_{1}, \cdots\right)$ be a doubly infinite sequence of independent identically distributed random variables. Let $T$ be the translation operator defined on $\eta$ by

$$
T_{\eta}=\eta^{\prime}=\left(\cdots, \eta_{0}, \eta_{1}, \eta_{2}, \cdots\right) .
$$

Let $B$ be the Borel field generated by $\eta$ and let $g=g(\eta)$ be a function defined on $B$ such that $E\left\{|g|^{2}\right\}<\infty$.

Given any such function $g$, we define a stationary process $\left\{X_{n}\right\}$ where

$$
X_{n}=g\left(T^{n} \eta\right), \quad n=0, \quad \pm 1, \cdots .
$$

We assume without loss of generality that $E\left\{X_{n}\right\} \equiv 0$. Let $r_{s}=E\left\{X_{n} X_{n+s}\right\}$. Then $r_{s}=\int_{-\pi}^{\pi} e^{i s \lambda} d F(\lambda)$, where $F(\lambda)$ is the spectral distribution function of the process. In $\$ 3$ the spectral distribution function of any process of the form (2.2) is shown to be absolutely continuous. Finally it is shown in $\S 4$ that under some additional assumptions on the moment structure of the process the central limit theorem is applicable.

3. Absolute continuity of the spectral distribution function. In this section we prove

Theorem I. If $F(\lambda)$ is the spectral distribution function of a process of the form (2.2), $F(\lambda)$ is absolutely continuous.

Proof. In proving the theorem we assume that each $\eta_{j}$ is uniformly distributed on the unit interval. It is easily seen that this may be done without loss of generality. For if $\xi$ is uniformly distributed on the unit interval, we can always construct a monotone function $\varphi(\xi)$ such that the distribution of $\varphi(\xi)$ is the same as that of any $\eta_{i}$, and consequently as far as the probability structure of the process $\left\{X_{n}\right\}$ is concerned, we may assume that each $\eta_{i}$ is uniformly distributed $[0,1]$.

Received May 29, 1956; in revised form, November 19, 1956. In this work, the authors were sponsored by the Office of Ordnance Research, U. S. Army, under Contract No. DA33-008-ORD-965, and the Office of Naval Research under Contract No. Nonr-908(10). 
For each integer $n$ define a process $\left\{X_{n, m}\right\}, m=1,2, \cdots$, by

$$
X_{n, m}=E\left\{X_{n} \mid \eta_{n-m+1}, \cdots, \eta_{n+m-1}\right\} .
$$

It is easily verified that for $n$ fixed, the process $\left\{X_{n, m}\right\}$ is a martingale. Furthermore $E\left\{\left|X_{n, m}\right|^{2}\right\} \leq E\left\{\left|X_{n}\right|^{2}\right\}=E\left\{|g(\eta)|^{2}\right\}<\infty$. It then follows from well-known theorems on martingales that $X_{n, m}$ converges in mean square and with probability one as $m$ approaches infinity. To see that $\lim _{m \rightarrow \infty} X_{n, m}=X_{n}$, note that $X_{n, m}$ is the projection of $X_{n}$ on the space generated by all measurable functions of $\eta_{n-m+1}, \cdots, \eta_{n+m-1}$. Since $X_{n}$ lies in the space generated by the entire $\eta$ sequence, it follows that $\lim _{m \rightarrow \infty} E\left\{\left|X_{n}-X_{n, m}\right|^{2}\right\}=0$. (See e.g. Doob [1;331, Theorem 4.3].

Let $\theta_{j}(u) ; j=0,1, \cdots$ be an orthonormal sequence of polynomials where $\theta_{j}(u)$ is of degree $j$ on $[0,1]$ i.e., $\int_{0}^{1} \theta_{j}(u) \theta_{k}(u) d u=\delta_{j k}$ where $\delta_{j k}$ is the Kronecker delta. The functions $\left\{\theta_{i}(u)\right\}$ are complete in $L^{2}[0,1]$. Let $\left(u_{1}, \cdots, u_{k}\right)$ be a point in the $k$-dimensional unit cube $U_{k}$. The sequence of products $\left\{\theta_{i_{1}}\left(u_{1}\right)\right.$ $\left.\cdots \theta_{i_{k}}\left(u_{k}\right)\right\}$ with $j_{1}, \cdots, j_{k}$ nonnegative integers is a complete orthonormal system for $L^{2}\left[U_{k}\right]$. To get an orthonormal system for the infinite-dimensional unit cube $U$ we take the function which is identically one and the system of products $\left\{\theta_{i_{1}}\left(u_{m_{1}}\right) \cdots \theta_{i_{k}}\left(u_{m_{k}}\right)\right\}$ where $m_{1}<m_{2}<\cdots<m_{k}$ and $k=1,2, \cdots$. This system is clearly orthonormal. Now let $k$ be an arbitrary positive integer. From the discussion above it follows that we can expand $X_{0, k}$ in the form

$$
X_{0, k}=\sum_{i=1}^{2 k-1} \sum_{-k+1 \leq m_{1}<\cdots<m_{j} \leq k-1} \sum_{n_{1}, \cdots, n_{j}=1}^{\infty} C\left(\bar{n}_{i}, \bar{m}_{i}\right) \varphi_{\bar{n}_{j}}\left(\eta_{\bar{m}_{j}}\right)
$$

where $\bar{m}_{j}=\left(m_{1}, \cdots, m_{j}\right), \bar{n}_{i}=\left(n_{1}, \cdots, n_{j}\right)$, and

$\varphi_{\bar{n}_{j}}\left(\eta_{\bar{m}_{j}}\right)=\theta_{n_{1}}\left(\eta_{m_{1}}\right) \cdots \theta_{n_{j}}\left(\eta_{m_{i}}\right)$. Since $E\left\{\left|X_{0, k}\right|^{2}\right\} \leq E\left\{\left|X_{0}\right|^{2}\right\}<\infty$

it follows that

$$
\sum_{j=1}^{\infty} \sum_{m_{1}<m_{2}<\cdots<m_{i}} \sum_{n_{1}}^{\infty}, \cdots, n_{i=1} C\left(\bar{n}_{i}, \bar{m}_{i}\right)^{2}<\infty .
$$

Now $X_{0}=\lim _{k \rightarrow \infty} X_{0, k}$ with probability one so that

$$
X_{0}=\sum_{j=1}^{\infty} \sum_{m_{1}<\cdots<m_{i}} \sum_{n_{1}}, \sum_{\cdots, n_{j}=1}^{\infty} C\left(\bar{n}_{j}, \bar{m}_{j}\right) \varphi_{\bar{n}_{j}}\left(\eta_{\bar{m}_{j}}\right)
$$

with probability one Similarly we have

$$
X_{n}=\sum_{j=1}^{\infty} \sum_{m_{1}<\cdots<m_{j} n_{1}} \sum_{\cdots, n_{j}=1}^{\infty} C\left(\bar{n}_{j}, \bar{m}_{i}-n\right) \varphi_{\bar{n}_{j}}\left(\eta_{\bar{m}_{j}}\right)
$$

where $\bar{m}_{j}-n=\left(m_{1}-n, \cdots, m_{j}-n\right)$.

Now let $k$ be a positive integer, $\bar{n}=\left(n_{1}, \cdots, n_{k}\right)$ be a $k$-tuplet of positive integers, and $\bar{m}=\left(m_{1}, \cdots, m_{k}\right)$ be a $k$-tuplet of integers with $m_{1}<\cdots<m_{k}$. 
Given any integer $t$, we define $X_{t}(\bar{m}, \bar{n})$ by

$$
X_{t}(\bar{m}, \bar{n})=\sum_{i=-\infty}^{\infty} C(\bar{n}, \bar{m}+j-t) \varphi_{\bar{n}}\left(\eta_{\bar{m}+j}\right)
$$

where $\bar{m}+l=\left(m_{1}+l, \cdots, m_{k}+l\right)$ for any integer $l . X_{t}(\bar{m}, \bar{n})$ is a moving average, and such processes are known to have an absolutely continuous spectrum $[1 ; 499]$. Now let $\left(\bar{m}^{\prime}, \bar{n}^{\prime}\right)$ be different from $(\bar{m}, \bar{n})$ in the sense that $\bar{n} \neq \bar{n}^{\prime}$ or that $\bar{m}-\bar{m}^{\prime}$ is not a vector with equal coordinates. Then it is easily seen that the processes $X_{t}(\bar{m}, \bar{n})$ and $X_{t}\left(\bar{m}^{\prime}, \bar{n}^{\prime}\right)$ are mutually orthogonal. There is a denumerable collection of such distinct processes and we enumerate them. Let $\left\{X_{t}^{(i)}\right\}$ be the $i$-th process and let $f^{(i)}(\lambda)$ be its spectral density. Consider the process $\sum_{i=1}^{n} X_{t}^{(i)}$ and let $f_{n}(\lambda)$ be its spectral density. From the mutual orthogonality of the processes $\left\{X_{t}^{(i)}\right\}$ it follows that $f_{n}(\lambda)=\sum_{i=1}^{n} f^{(i)}(\lambda)$. Since the $f^{(i)}(\lambda)$ are nonnegative, $f_{n}(\lambda)$ is non-decreasing in $n$. Let $f(\lambda)=$ $\lim _{n \rightarrow \infty} f_{n}(\lambda)$. Then we have

$$
\begin{aligned}
\int_{-\pi}^{\pi} f(\lambda) d \lambda=\int_{-\pi}^{\pi} \lim _{n \rightarrow \infty} f_{n}(\lambda) d \lambda & \leq \lim _{n \rightarrow \infty} \int_{-\pi}^{\pi} f_{n}(\lambda) d \lambda \\
= & \lim _{n \rightarrow \infty} E\left\{\left|\sum_{i=1}^{n} X_{t}^{(i)}\right|^{2}\right\}=E\left\{|g|^{2}\right\}<\infty .
\end{aligned}
$$

Hence $f(\lambda)$ is almost everywhere finite and integrable. An easy computation shows that $f(\lambda)$ is the spectral density of the process $\left\{X_{t}\right\}$ and the theorem is proved.

It follows from the theorem just proved that if $h$ is any square integrable function defined on the probability space generated by $X=\left(\cdots, X_{-1}, X_{0}\right.$, $\left.X_{1}, \cdots\right)$ and if $\left\{U_{n}\right\}$ is the process defined by $U_{n}=h\left(T^{n} X\right)$ then the process $\left\{U_{n}\right\}$ also has an absolutely continuous spectrum. It would be of some interest to determine if the converse of this proposition is also true. That is, if $\left\{X_{n}\right\}$ is strictly stationary and if $\left\{X_{n}\right\}$ and any process $\left\{U_{n}\right\}$ obtained from $\left\{X_{n}\right\}$ in the manner indicated above has an absolutely continuous spectrum, does it follow that $X_{n}$ is of the form $g\left(T^{n} \eta\right)$ where $g$ is square integrable and $\eta=(\cdots$, $\left.\eta_{-1}, \eta_{0}, \eta_{1}, \cdots\right)$ is a doubly infinite sequence of independent identically distributed random variables?

4. The central limit theorem. In this section it is shown that under some additional moment conditions, the processes we have discussed satisfy the central limit theorem. As before let $X_{n, k}=E\left\{X_{n} \mid \eta_{n-k+1}, \cdots, \eta_{n+k-1}\right\}$ when $k=1,2, \cdots$, and let $X_{n, 0}=0$. Furthermore, let $V_{n, k}=X_{n, k+1}-X_{n, k}$, and for any integer $s$ let $a_{s, k, k^{\prime}}=E\left\{V_{n, k} V_{n+s, k^{\prime}}\right\}$. Then we have

Theorem 2. If

$$
E\left\{\left|X_{n}\right|^{\alpha}\right\}<\infty \text { for some } \alpha>2,
$$




$$
\sum_{s=-\infty}^{\infty} \sum_{k, k^{\prime}=1}^{\infty}\left|a_{s, k, k^{\prime}}\right|<\infty
$$

and

$$
\sum_{s=-\infty}^{\infty} \sum_{k, k^{\prime}=1}^{\infty} a_{s, k, k^{\prime}} \neq 0
$$

then $N^{-\frac{1}{2}} \sum_{i=1}^{N} X_{i}$ is asymptotically normal with mean zero and variance $2 \pi f(0)$, where $f(\lambda)$ is the spectral density of the process $\left\{X_{n}\right\}$.

Proof. For fixed $k$ it can easily be verified that the process $\left\{X_{n, k}\right\}$ is a stationary $(2 k-1)$-dependent process. Marsaglia [2] has given conditions under which such a process satisfies the central limit theorem. Let $s_{N, k}^{2}=$ $E\left\{\left|\sum_{i=1}^{N} X_{i, k}\right|^{2}\right\}$. Then we can state Marsaglia's conditions in the way we shall use them:

$$
\begin{gathered}
E\left\{\left|X_{n, k}\right|^{2}\right\}<B<\infty, \quad k=1,2, \cdots, \\
\frac{N}{s_{N, k}^{2}}<B, \quad N=1,2, \cdots, \\
\lim _{N \rightarrow \infty} \frac{N^{1 / \alpha} E\left\{\left|X_{n, k}\right|^{\alpha}\right\}^{1 / \alpha}}{s_{N, k}}=0, \text { for some } \alpha>2 .
\end{gathered}
$$

We first show that these conditions are satisfied for $k$ sufficiently large. Condition (4.4) follows immediately from the fact that $E\left\{\left|X_{n, k}\right|^{2}\right\} \leq E\left\{|g|^{2}\right\}<\infty$. To prove (4.5) we write

$$
\begin{aligned}
\frac{s_{N, k}^{2}}{N} & =\frac{1}{N} E\left\{\left(\sum_{i=1}^{N} \sum_{i=0}^{k-1} V_{i, i}\right)^{2}\right\} \\
& =\frac{1}{N} \sum_{i, i^{\prime}=0}^{k-1} \sum_{i, i^{\prime}=1}^{N} a_{i^{\prime}-i, i, i^{\prime}} \\
& =\frac{1}{N} \sum_{i, i^{\prime}=0}^{k-1} \sum_{u=-N+1}^{N-1} a_{u, i, i^{\prime}}(N-|u|),
\end{aligned}
$$

where $u=i^{\prime}-i$. Hence

$$
\lim _{N \rightarrow \infty} \frac{s_{N, k}^{2}}{N}=\sum_{j, j^{\prime}=0}^{k-1} \sum_{u=-\infty}^{\infty} a_{u, i, i^{\prime}} .
$$

Given hypothesis (4.3) we can clearly choose $k$ so large that (4.5) is satisfied. Jensen's inequality is used to obtain $E\left\{\left|X_{n k}\right|^{\alpha}\right\} \leq E\left\{\left|X_{n}\right|^{\alpha}\right\}$ for all $k$. Condition (4.6) follows immediately.

Now let $r_{i}=E\left\{X_{n} X_{n+i}\right\}, r_{i, k}=E\left\{X_{n, k} X_{n+j, k}\right\}$, and let $f_{k}(\lambda)$ be the spectral density of the process $\left\{X_{n, k}\right\}$. Then

$$
f(\lambda)=\frac{1}{2 \pi} \sum_{j=-\infty}^{\infty} r_{j} e^{-i j \lambda}
$$


A CLASS OF STATIONARY PROCESSES

and

$$
f_{k}(\lambda)=\frac{1}{2 \pi} \sum_{j=-\infty}^{\infty} r_{i, k} e^{-i j \lambda}
$$

Hence

$$
\left|f_{k}(\lambda)-f(\lambda)\right| \leq \frac{1}{2 \pi} \sum_{j=-\infty}^{\infty}\left|r_{i, k}-r_{i}\right| .
$$

For each $j$ we have $\lim _{k \rightarrow \infty} r_{i, k}=r_{i}$. Furthermore it is easily established that

$$
\sum_{i \geq i_{0}}\left|r_{i}\right| \leq \sum_{i \geq i_{0}} \sum_{i, i^{\prime}=0}^{\infty}\left|a_{j, i, i^{\prime}}\right|
$$

and

$$
\sum_{j \geq i_{0}}\left|r_{i, k}\right| \leq \sum_{i \geq i_{0}} \sum_{i, i^{\prime}=0}^{\infty}\left|a_{i, i, i^{\prime}}\right| \text { uniformly in } k .
$$

Applying hypothesis (4.2) we see that

$$
\lim _{k \rightarrow \infty} \frac{1}{2 \pi} \sum_{i=-\infty}^{\infty}\left|r_{i, k}-r_{i}\right|=0 .
$$

Thus it is clearly seen that $\lim _{k \rightarrow \infty} f_{k}(\lambda)=f(\lambda)$ uniformly in $\lambda$. Since

$$
f(0)=\frac{1}{2 \pi} \sum_{j=-\infty}^{\infty} r_{j}=\frac{1}{2 \pi} \sum_{j=-\infty}^{\infty} \sum_{k, k^{\prime}=0}^{\infty} a_{i, k, k^{\prime}}>0,
$$

we may choose $k$ so large that $f_{k}(0)>0$ and so that Marsaglia's theorem applies.

Then

$$
\lim _{N \rightarrow \infty} P\left\{\left(2 \pi N f_{k}(0)\right)^{-\frac{1}{2}} \sum_{i=1}^{N} X_{i, k} \leq \alpha\right\}=(2 \pi)^{-\frac{1}{2}} \int_{-\infty}^{\alpha} e^{-u^{2} / 2} d u
$$

Now

$$
\begin{aligned}
E\left\{\left|\left(N f_{k}(0)\right)^{-\frac{1}{2}} \sum_{i=1}^{N}\left(X_{i}-X_{i, k}\right)\right|^{2}\right\} & =\frac{1}{N f_{k}(0)} E\left\{\left|\sum_{i=1}^{N} \sum_{j=k+1}^{\infty} V_{n, i}\right|^{2}\right\} \\
& \leq \frac{1}{N f_{k}(0)} \sum_{i, i^{\prime}=1}^{N} \sum_{i, i^{\prime}=k+1}^{\infty}\left|a_{i^{\prime}-i^{\prime}, i^{\prime}}\right| \\
& =\frac{1}{f_{k}(0)} \sum_{j, j^{\prime}=k+1}^{\infty} \sum_{u=-N}^{N}\left|a_{u, i^{\prime}, j^{\prime}}\right|\left(1-\frac{|u|}{N}\right)
\end{aligned}
$$

where $u=i^{\prime}-i$. On letting $N$ approach infinity in the last sum we obtain

$$
\frac{1}{f_{k}(0)} \sum_{j, j^{\prime}=k+1}^{\infty} \sum_{u=-\infty}^{\infty}\left|a_{u, i, j},\right|
$$

Clearly we may make this sum arbitrarily small by choosing $k$ large enough. 
Since $\lim _{k \rightarrow \infty} f_{k}(0)=f(0)$ we obtain

$$
\lim _{N \rightarrow \infty} P\left\{(2 \pi N f(0))^{-\frac{1}{2}} \sum_{i=1}^{N} X_{i} \leq \alpha\right\}=(2 \pi)^{-\frac{1}{2}} \int_{-\infty}^{\alpha} e^{-u^{2} / 2} d u
$$

which is the desired result.

\section{References}

1. J. L. Dоoв, Stochastic Processes, New York, 1953.

2. G. Marsaglia, Iterated limits and the central limit theorem for dependent variables, Proceedings of the American Mathematical Society, vol. 5(1954), pp. 987-991.

\section{INDIANA UNIVERSITY}

